\title{
HgCdTe quantum wells grown by molecular beam epitaxy
}

\author{
S.A. Dvoretsky, D.G. Ikusov, Z.D. Kvon, N.N. Mikhailov, V.G. Remesnik, \\ R.N. Smirnov, Yu.G. Sidorov, V.A. Shvets \\ A.V. Rzhanov Institute of Semiconductor Physics of the Siberian Branch of Russian Academy of Sciences \\ 13, Acad. Lavrent'ev Ave., 630090 Novosibirsk, Russia \\ Phone, fax: +7-383-330-49-67. \\ E-mail:dvor@isp.nsc.ru
}

\begin{abstract}
Cd}_{\mathrm{x}} \mathrm{Hg}_{1-\mathrm{x}}$ Te-based $(x=0-0.25)$ quantum wells (QWs) of $8-22 \mathrm{~nm}$ in thickness were grown on (013) $\mathrm{CdTe} / \mathrm{ZnTe} / \mathrm{GaAs}$ substrates by molecular beam epitaxy. The composition and thickness $(d)$ of wide-gap layers (spacers) were $x \sim 0.7$ mol.frac. and $d \sim 35 \mathrm{~nm}$, respectively, at both sides of the quantum well. The thickness and composition of epilayers during the growth were controlled by ellipsometry in situ. It was shown that the accuracy of thickness and composition were $\Delta x= \pm 0.002, \Delta d=$ $\pm 0.5 \mathrm{~nm}$. The central part of spacers (10 nm thick) was doped by indium up to a carrier concentration of $\sim 10^{15} \mathrm{~cm}^{-3}$. A CdTe cap layer $40 \mathrm{~nm}$ in thickness was grown to protect QW. The compositions of the spacer and QWs were determined by measuring the $E_{1}$ and $E_{1}+\Delta_{1}$ peaks in reflection spectra using layer-by-layer chemical etching. The galvanomagnetic investigations (the range of magnetic fields was $0-13 \mathrm{~T}$ ) of the grown $\mathrm{QW}$ showed the presence of a $2 \mathrm{D}$ electron gas in all the samples. The $2 \mathrm{D}$ electron mobility $\mu_{e}=(2.4-3.5) \times 10^{5} \mathrm{~cm}^{2} /(\mathrm{V} \cdot \mathrm{s})$ for the concentrations $N=(1.5-3) \times 10^{11} \mathrm{~cm}^{-2}(x<0.11)$ that confirms a high quality of the grown QWs.
\end{abstract}

Keywords: molecular beam epitaxy, mercury cadmium telluride $\mathrm{HgCdTe}$, quantum well, ellipsometry, infrared spectroscopy, quantum Hall effect.

Manuscript received 20.11.07; accepted for publication 19.12.07; published online 31.01.08.

\section{Introduction}

The interest to nanostructures based on II-VI narrow-gap semiconductors containing mercury (such as $\mathrm{HgTe} / \mathrm{CdTe}$ superlattices) was due to their advantages in comparison with mercury cadmium telluride (MCT) alloys on the fabrication of high-quality multiple-unit infrared (IR) photodetectors firstly from the point of view of uniformity of a spectral response [1]. However, the first growth experimental results did not lead to high-quality nanostructures [2]. The development of molecular beam epitaxy (MBE) technique in last years allows one to grow nanostructures suitable for the development and fundamental investigations of devices. The authors in [3] demonstrated the growth of doped $\mathrm{HgTe} / \mathrm{HgCdTe}$ superlattices which were used for the fabrication of very long wavelength IR photodetectors (cut-off wavelength over $20 \mu \mathrm{m}$ ). Microcavity $\mathrm{Cd}_{0.65} \mathrm{Hg}_{0.35} \mathrm{Te} / \mathrm{Cd}_{0.36} \mathrm{Hg}_{0.64} \mathrm{Te}$ $(350 \mathrm{~nm} / 250 \mathrm{~nm}$ in thickness) structures were grown by $\mathrm{MBE}$ and used to fabricate 3-3.5 $\mu \mathrm{m}$ IR photoemitters [4].
The fundamental characteristics of HgTe-based quantum wells (QWs) were intensively studied (see [58]). It was shown that the electron effective mass in HgTe-based QWs is lower than that in QWs of III-V compounds. This leads to a wider splitting of Landau levels, weaker electron localization, increasing the amplitude of Shubnikov-de Haas oscillations, and increasing the Rashba effect [5]. HgTe-based QWs with thicknesses over than $5 \mathrm{~nm}$ are characterized by the inverted conduction band (the band of light holes becomes similar to the conduction band) that is undoubtedly interesting for studying the electron behavior in such a system. It is interesting also the behavior of electrons in $\mathrm{HgCdTe}$-based QWs at small Cd mole fractions. From fundamental considerations, this should lead to decreasing the band gap (up to a zero-gap semiconductor) with increase in the $\mathrm{Cd}$ content and, as a consequence, to changing the thickness, at which the band inversion occurs. So it is of interest to investigate $\mathrm{HgCdTe}$-based QWs at increasing the Cd content beyond a zero-gap semiconductor, at which no band inversion occurs. 
At the growth of nanostructures, the precise control over the MCT composition and thickness is very important. However, there are no data in the literature about providing such a control at the growth of QWs.

The layers thickness control at the growth of QWs on the basis of III-V compounds in Si-Ge system is carried out by measuring the reflection high energy electron diffraction (RHEED) oscillations. However, using RHEED at the growth of HgCdTe-based QWs is impossible for two reasons. First, the electron beam with an energy over than $15 \mathrm{keV}$ essentially influences the growth process, which leads to variations in the surface morphology and MCT composition. Second, the RHEED oscillations are observed only during the growth on singular (001) orientations. However, because of technological reasons, the growth of MCT alloys is usually carried out on the vicinal (112)B, (013) orientations of substrates.

We showed a very effective use of ellipsometry for the growth of MCT heteroepitaxial structures (HS). This technique allows one to provide a high-precision control in situ of the MCT composition, thickness, and growth rates. This allows one to grow MCT HS with a special MCT composition profile throughout the thickness for different IR detectors [9-11]. The use of ellipsometry for the control over the nanolayers growth was firstly shown in [12]. The author in [13] presented the calculations of ellipsometric parameters changing at the $\mathrm{HgTe} / \mathrm{CdTe}$ superlattices growth that ensures their possible effective use in practice. The ellipsometry control over the growth of MCT-based potential barriers, wells, and periodical structures with $100 \mathrm{~nm}$ in thickness was carried out in [14]. There, the possibility to grow nanostructures ellipsometrically controlled in situ at growth rates up to $3.5 \mu \mathrm{m} / \mathrm{h}$ was demonstrated. The accuracies of the determination of the MCT composition and thickness were $\Delta x \sim \pm 0.002 \mathrm{~mol}$.frac. and $\Delta d \sim 1$ monolayer, respectively.

The aim of the present paper is to present the results of the growth of $\mathrm{Hg}_{1-\mathrm{x}} \mathrm{Cd}_{\mathrm{x}}$ Te-based QWs $(x=$ $0-0.25)$ with precise ellipsometric control of the MCT composition and thickness in situ on (013) $\mathrm{CdTe} / \mathrm{ZnTeGaAs}$ substrates and their magnetogalvanic investigation.

\section{Experimental results and discussion}

An MCT-based QW structure is shown schematically in Fig. 1. The QW growth was carried out on the MBE equipment of the "Ob-M"-type [11]. The buffer layers of $\mathrm{ZnTe}(0.3 \mu \mathrm{m})$ and sequence $\mathrm{CdTe}(5-7 \mu \mathrm{m})$ were grown on the atomic clean surface of (013) GaAs substrate preparing by chemical etching and thermal annealing in the As flux in an ultra vacuum chamber [15]. Then the growth of an MCT-based $(x=0-0.25)$ QW with a spacer $(x \sim 0.7)$ was carried out on the CdTe buffer layer surface at a temperature of $180-190{ }^{\circ} \mathrm{C}$. The doping of the central part of a spacer up to the carrier

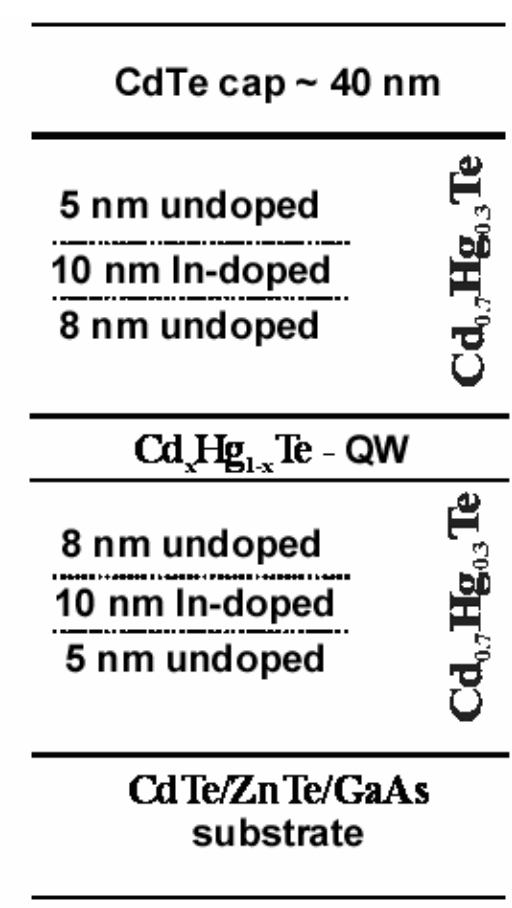

Fig. 1. Scheme of $\mathrm{a} \mathrm{Cd}_{\mathrm{x}} \mathrm{Hg}_{1-\mathrm{x}} \mathrm{Te}$-based quantum well.

concentration $10^{15} \mathrm{~cm}^{-3}$ was realized in situ with the use of an In conventional source [16]. The growth rate was $0.15 \mu \mathrm{m} / \mathrm{h}$. The MCT composition during the growth was varied by a cadmium flux. An ultra fast single-beam $(\lambda=0.6328)$ automatic ellipsometer LEF-755 (UFE) based on the original static scheme [17] was used for the control over the epilayer buffer thickness and the MCT composition and thickness.

At the epitaxial growth of a layer on the substrate with different optical constants, the decaying oscillations of the ellipsometric parameters $\psi$ and $\Delta$ were observed. The number of oscillations depends on the absorption coefficient of a growing layer and decreases with increase in its value. The oscillation amplitude is determined by the difference of the absorption coefficients of the substrate and the growing layer. One period of oscillations corresponds to a 100-nm MCT layer. At the growth of multilayer structures with thicknesses lower than $100 \mathrm{~nm}$, one should observe sectionally smooth curves which correspond to the growth of MCT layers with constant composition. The length of a sectionally smooth curve corresponds to the thickness of a growing layer.

The evolution of the ellipsometric parameters $\Delta$ and $\psi$ during the growth of HgTe-based QWs is shown in Fig. 2. It is represented as a sectionally smooth curve in the $\psi-\Delta$ plane. Smooth sections correspond to the constant MCT composition of layers. Their length determines the layer thickness. The initial point $\mathrm{O}$ corresponds to the ellipsometric parameters of the CdTe surface. The fabrication of a QW begins from the growth 


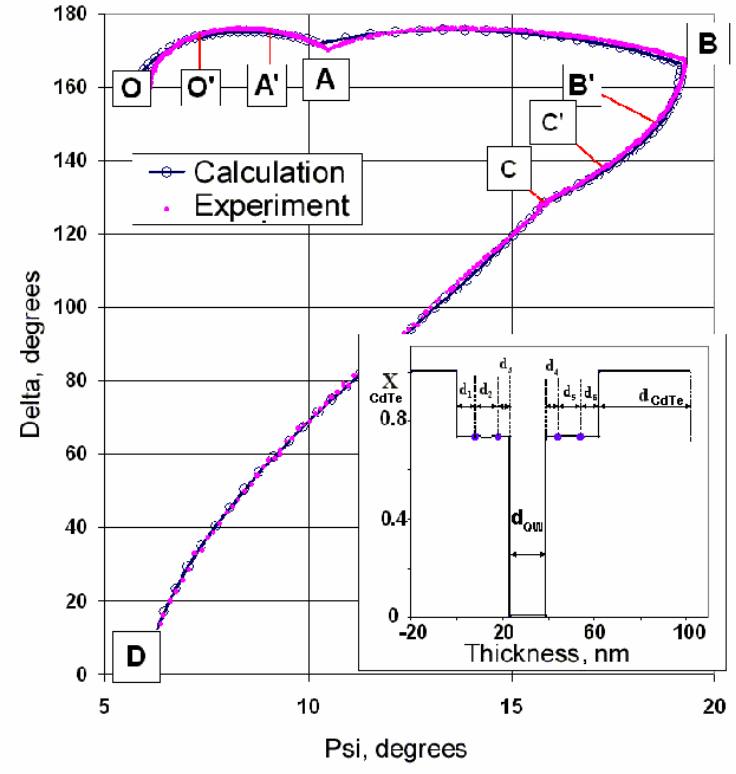

Fig. 2. Evolution of the ellipsometric parameters $\Delta$ and $\psi$ during the growth of a HgTe-based QW. The points at breaks of the curve (O-C) correspond to the initial stage of growth of the following QW layer. Dots - experimental data; solid line and circles (through $1 \mathrm{~nm}$ ) - calculated data. In the inset: the MCT composition versus the thickness.

of a wide-gap layer $(x \sim 0.7)$ (curve O-A) after the opening of the tellurium and cadmium source shutters. The doping of the central part of this layer is carried out by indium after the opening at $\mathrm{O}^{\prime}$ and the closing at $\mathrm{A}^{\prime}$ of the indium source shutter. Then the growth of a widegap layer is continued up to point $\mathrm{A}$. In the insert, the sectors $\mathrm{O}-\mathrm{O}^{\prime}, \mathrm{O}^{\prime}-\mathrm{A}^{\prime}$, and $\mathrm{A}^{\prime}-\mathrm{A}$ correspond to the undoped wide-gap layer with thickness $d_{1}$, the In-doped wide-gap layer with thickness $d_{2}$, and the undoped widegap layer with thickness $d_{3}$. After the closing of the cadmium source shutter, the $\mathrm{HgTe}$ layer is growing between A-B points of the curve with thickness $d_{\mathrm{QW}}$. The wide-gap layer (see B-C curve) is growing in manner that is analogous to the growth of the first widegap layer (O-A curve) after the opening of the cadmium source shutter. The thicknesses $d_{4}, d_{6}$, and $d_{5}$ correspond to undoped and In-doped wide-gap layers. The grown QW structure is covered by a CdTe cap layer with $d_{\text {CdTe }} \sim 40 \mathrm{~nm}$.

The dots on the curve in Fig. 2 are experimental data which were measured at 1s-intervals. The circles correspond to the values of ellipsometric parameters calculated at $1-\mathrm{nm}$ intervals. The calculations were carried out by using the one-layer model [18] analogously to [19]. The calibration curve and the optical constants for calculations were taken for different MCT compositions from $[20,21]$. To improve the comparison of the experimental and calculated data, it was necessary to know the optical constants of the substrate and the growing layers at incident light wavelengths and the growth temperature with high accuracy. The MCT optical constants for different compositions were obtained from the spectral measurements at the room or a lower temperature $[22,23]$. But these data cannot be used for calculating the variation of ellipsometric parameters during the growth of a QW which occurs at much higher temperatures. Thus, we used the dependences of the optical constants $[n(x)$ and $k(x)]$ on the MCT composition which were measured at room temperature [20] and then calculated them for temperatures of the growth using the experimentally determined thermooptical coefficients. The experimental data are in good agreement with the results of calculations (Fig. 2) within this procedure.

The procedure of determination of the MCT composition and the layer thickness for the growth of a wide-gap layer on the CdTe surface is shown in more details in Fig. 3. The point $\mathrm{O}$ corresponds to the ellipsometric parameters of the CdTe surface. After the start of the growth, a smooth variation of the ellipsometric parameters $\psi$ and $\Delta$ is observed (O-A sector). After point $\mathrm{A}$, the growth of a $\mathrm{HgTe}$ layer is running. Lines $1-3$ are the calculated data for the growth of MCT layers with different MCT compositions $x=0.75$ (1), 0.735 (2), and 0.72 (3) and the following parameters: the angle of incident light $-67.9^{\circ} \pm 0.05^{\circ}$; $n_{\mathrm{CdTe}}=3.003 ; \quad k_{\mathrm{CdTe}}=0.204 ; n_{\mathrm{HgTe}}=4.08$, and $k_{\mathrm{HgTe}}=1.16$. These parameters were determined by measuring the ellipsometric parameters during the growth of an epilayer with constant MCT composition. The thickness of the growing wide-gap layer is determined by the length of O-A curve. The thick transverse lines to O-A curve mean the line of equal thickness for different MCT compositions. It is clear that the accuracy of the MCT composition and the layer thickness is determined by the coincidence of experimental and calculated curves and the accuracy of

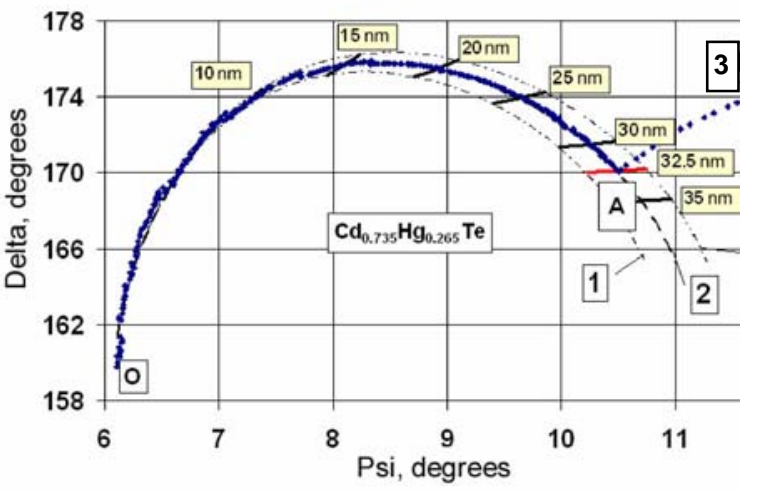

Fig. 3. Evolution of ellipsomentric parameters $\Delta$ and $\psi$ at the wide-gap layer growth on the CdTe surface. Rhombs experimental data. Lines 1-3 - calculated MCT composition $\left(1-x_{\mathrm{CdTe}}=0.75,2-0.735\right.$, and $\left.3-0.72\right)$ The transverse line to O-A - lines of equal thicknesses for different MVT compositions. 
the position of the inflection point. For the data in Fig. 2, the MCT composition was determined using the abovementioned procedure as $x=0.735 \pm 0.002$. The upper bound of the thickness determination accuracy does not exceed $0.5 \mathrm{~nm}$, i.e., 1 monolayer. In an analogous manner, we performed the analysis of other parts of the curve in Fig. 2.

Thus, we determined the MCT compositions and the thickneses of a HgTe-base QW. The determination accuracies of the MCT composition and the thickness were $\Delta x \sim 0.002$ and $0.5 \mathrm{~nm}$, respectively.

The MCT compositions and thicknesses of layers of seven $\mathrm{HgCdTe}$-based QWs are given in Table.

The MCT composition distribution throughout the thickness of a $\mathrm{HgCdTe}$-based QW was measured by reflection spectra in the range $1.5-6 \mathrm{eV}$ with layer-bylayer chemical etching in a $0.05 \% \mathrm{Br}-\mathrm{HBr}$ solution.

The thickness of a layer removed by chemical etching was determined by the shifts of the interference maxima of transmission spectra in the range $1600-$ $2300 \mathrm{~cm}^{-1}$. The measurements of the transmission spectra were carried out using an IR Fourierspectrometer "Infralum FT-801" $(2-25 \mu \mathrm{m})$. The positions of interference maxima are described by the relation $2 n d=m \times 10^{4} / \mathrm{v}$, where $n-$ refraction index, $d-$ thickness in $\mu \mathrm{m}, m$ - maximum order, and $v-$ wavenumber in $\mathrm{cm}^{-1}$. It was easy to evaluate that a $1-\mathrm{nm}$ variation of the CdTe thickness leads to a $0.3-\mathrm{cm}^{-1}$ shift of interferential maxima for $5.5-\mu \mathrm{m} \mathrm{CdTe}$, and the refraction index $n_{\mathrm{CdTe}}=2.8 \quad\left(v=1780 \mathrm{~cm}^{-1}\right)$. The accuracy of the determination of a position of interference maxima with the use of an "Infralum FT-801" is lower than $0.01 \mathrm{~cm}^{-1}$. Fig. 4 shows the shifts of interference maxima during the removal of a layer of $5486.8 \mathrm{~nm}$ in thickness at QW N 221 (see Table) under chemical etching. After the removal of 31.9-nm layers, the interference maxima shift to thicknesses of 5454.9 and $5430.0 \mathrm{~nm}$, respectively. The thickness determination accuracy is lower than $0.1 \mathrm{~nm}$.

The measurement of the reflection spectra of narrow-gap semiconductors (1.5-6 eV range) allows one to determine the shape of $E_{1}$ and $E_{1}+\Delta_{1}$ peaks and their energy positions. These peaks are related to the critical points of the density of states for direct band gap transitions at energies over the band gap. For MCT compounds, the positions of peaks depend on the MCT composition, which allows one to determine $x$ with accuracy $\sim 0.5 \%$ [24]. Some formulas for the dependence of $E_{1}$ and $E_{1}+\Delta_{1}$ peaks on the MCT composition are available in the literature $[24,25]$. In the present work, we have measured the reflection spectra at the room temperature to determine the position of the $E_{1}$ and $E_{1}+\Delta_{1}$ peaks for MCT epitaxial layers grown by MBE with a constant MCT composition $(x=0-1)$. These data are described by the following expressions:

$E_{1}=2.0944+0.5869 x+0.6295 x^{2}$,

$E_{1}+\Delta_{1}=2.7441+0.4895 x+0.6895 x^{2}$.

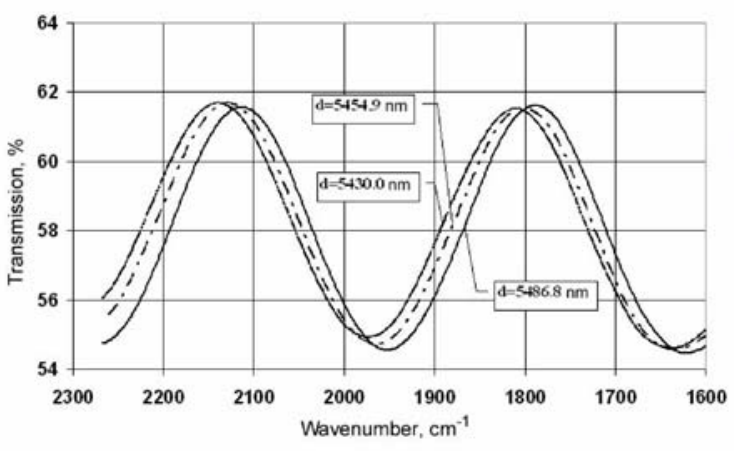

Fig. 4. Position of inference maxima during chemical etching of QW N 221 with an initial thickness of $5486.8 \mathrm{~nm}$. The numbers show the thicknesses measured after a sequence of chemical etchings.

These dependences were used for the structure composition determination.

The reflection spectra of HgCdTe-based QWs were measured at room temperature using a "SPECORD UVVIS" equipped by reflection attachments alternating with layer-by-layer chemical etching in a $0.05 \% \mathrm{Br}-\mathrm{HBr}$ solution. The reflection from an $\mathrm{Al}$ mirror was used as the reference signal. Fig. 5 shows the reflection spectrum of QW N 221 of the initial surface (curve 1) and that after three steps of chemical etching (curves 2-4). The peak at $3.31 \mathrm{eV}$ (curve 1) corresponds to the $E_{1}$ transition in CdTe that is in agreement with [24] and with data for bulk CdTe (curve 1 '). It seems that the peak near $2.2 \mathrm{eV}$ is related to the interference of the incident light and the light reflected from QW interfaces. After removing a 32-nm layer (curve 2), the peaks at 2.75 and $3.37 \mathrm{eV}$ correspond to the $E_{1}$ and $E_{1}+\Delta_{1}$ transitions for the MCT composition $x \sim 0.67$. Then, after removing a 57-nm layer (curve 3 ), the reflection spectrum is essentially changed. The peak at the $3.0-$ 3.5-eV interval disappears. Apparently, the peaks at 2.11 and $2.76 \mathrm{eV}$ correspond to the $E_{1}$ and $E_{1}+\Delta_{1}$ transitions for the MCT composition $x \sim 0.03$. Finally, after removing a 89-nm layer (curve 4), peaks at 2.66 and $3.27 \mathrm{eV}$ correspond to the $E_{1}$ and $E_{1}+\Delta_{1}$ transitions for the MCT composition $x \sim 0.67$ that is similar to that observed on curve 2. After removing the $\mathrm{QW}$, the reflection spectrum corresponds to the buffer CdTe layer (curve $1^{\prime}$ ).

The results of measurements of the MCT composition by ellipsometry in situ and by reflection spectra with layer-by-layer chemical etching are in a good agreement. Really, we need to take into account the influence of thin layers with different compositions on the position of peaks because of the penetration of incident light through these layers and the interference effects.

For all the grown samples (Table, except for $\mathrm{N} 219$ ), the magnetransport measurements were carried out at temperatures of $1.6-4.2 \mathrm{~K}$ at a magnetic field up to $13 \mathrm{~T}$ using standard Hall bars $(50 \mu \mathrm{m}$ in width, and 
Table. Parameters of grown QW samples.

\begin{tabular}{|c|c|c|c|c|c|c|c|c|c|c|c|}
\hline \multirow{2}{*}{$\mathbf{N}$} & \multicolumn{3}{|c|}{$\begin{array}{l}\text { MCT composition of a } \\
\text { wide-gap layer, } x_{\mathrm{CdTe}}\end{array}$} & \multirow{2}{*}{$\begin{array}{c}\mathrm{MCT} \\
\text { composition } \\
\text { of QW, } x \\
\begin{array}{c}D_{\mathrm{QW}}, \\
\mathrm{nm}\end{array}\end{array}$} & \multicolumn{3}{|c|}{$\begin{array}{l}\text { MCT composition of a } \\
\text { wide-gap layer, } x_{e}\end{array}$} & \multirow{2}{*}{$\begin{array}{c}\text { Thickness } \\
\text { of a } \\
\text { CdTe cap } \\
\text { layer } \\
d_{\text {CdTe }}, \mathrm{nm}\end{array}$} & \multirow{2}{*}{$\begin{array}{c}\text { MCT } \\
\text { composition } \\
\text { of QW, } x \text {, } \\
\text { from } \\
\text { reflection } \\
\text { spectra }\end{array}$} & \multirow{2}{*}{$\begin{array}{c}\text { Carrier } \\
\text { concentra- } \\
\text { tion, } \\
10^{11} \mathrm{~cm}^{-2}\end{array}$} & \multirow{2}{*}{$\begin{array}{l}\text { Mobility, } \\
\mathrm{cm}^{2} /(\mathrm{V} \cdot \mathrm{s})\end{array}$} \\
\hline & $\begin{array}{l}d_{1}, \\
\mathrm{~nm}\end{array}$ & $d_{2}, \mathrm{~nm}$ & $d_{3}, \mathrm{~nm}$ & & $d_{4}, \mathrm{~nm}$ & $d_{5}, \mathrm{~nm}$ & $d_{6}, \mathrm{~nm}$ & & & & \\
\hline \multirow[t]{2}{*}{218} & \multicolumn{3}{|c|}{0.73} & 0.06 & \multicolumn{3}{|c|}{0.63} & \multirow[b]{2}{*}{34} & \multirow[b]{2}{*}{-} & \multirow[b]{2}{*}{2} & \multirow[b]{2}{*}{24} \\
\hline & 9 & 12.3 & 10.2 & 20.3 & 9.1 & 10.2 & 8.2 & & & & \\
\hline \multirow[t]{2}{*}{219} & \multicolumn{3}{|c|}{0.79} & 0.24 & \multicolumn{3}{|c|}{0.80} & \multirow[b]{2}{*}{34} & \multirow[b]{2}{*}{0.05} & \multirow[b]{2}{*}{ - } & \multirow[b]{2}{*}{-} \\
\hline & 8.4 & 14.3 & 8.8 & 12.5 & 7.5 & 14.7 & 4.8 & & & & \\
\hline \multirow[t]{2}{*}{220} & \multicolumn{3}{|c|}{0.70} & 0.16 & \multicolumn{3}{|c|}{0.7} & \multirow[b]{2}{*}{38} & \multirow[b]{2}{*}{-} & \multirow[b]{2}{*}{1.6} & \multirow[b]{2}{*}{8.6} \\
\hline & 8.8 & 10.9 & 9.8 & 10.5 & 10 & 12 & 7.9 & & & & \\
\hline \multirow[t]{2}{*}{221} & \multicolumn{3}{|c|}{0.72} & 0.11 & \multicolumn{3}{|c|}{0.69} & \multirow[b]{2}{*}{44} & \multirow[b]{2}{*}{0.03} & \multirow[b]{2}{*}{3} & \multirow[b]{2}{*}{30} \\
\hline & 6.1 & 13.9 & 11.4 & 16.2 & 12.4 & 15.6 & 5.0 & & & & \\
\hline 330 & & 0.68 & & 0.03 & & 0.66 & & & & & \\
\hline & 7.1 & 6.4 & 11.2 & 18.4 & 8.6 & 9.5 & 5.0 & 46 & 0.00 & 2.2 & 35 \\
\hline 331 & & 0.7 & & 0.06 & & 0.65 & & & & & \\
\hline & 8.6 & 12.8 & 11.3 & 21.7 & 8.0 & 10.0 & 4.8 & 39 & - & 1.5 & 24 \\
\hline
\end{tabular}

the $100-\mu \mathrm{m}$ interval between potentiometric contacts) fabricated by means of photolithography. Indium ohmic contacts were formed by thermal compression. The electron concentrations and mobilities determined from these measurements at $4.2 \mathrm{~K}$ are presented in Table. The high value of mobility $\mu_{e}=(2.4-3.5) \times 10^{5} \mathrm{~cm}^{2} /(\mathrm{V} \cdot \mathrm{s})$ for electron concentrations $N_{s}=(1.5-3) \times 10^{11} \mathrm{~cm}^{-2}$ reveals a high quality of grown QWs. The investigations of Hall structures made on different areas of the plate show identical results, which testifies to a high $2 \mathrm{D}$ uniformity of samples. The results obtained for samples N 218 and 331 confirm a high reproducibility of the QW growth technology. The identical mobility $\left(\mu_{e}=\right.$ $\left.2.4 \times 10^{5} \mathrm{~cm}^{2} /(\mathrm{V} \cdot \mathrm{s})\right)$ and close concentrations $\left(N_{s}=\right.$ $2 \times 10^{11} \mathrm{~cm}^{-2}$ and $N_{s}=1.5 \times 10^{11} \mathrm{~cm}^{-2}$ ) were obtained for QWs with similar compositions and thicknesses ( $x=0.06$ and $d \approx 21 \mathrm{~nm}$ ). The samples were grown on the same MBE system with a time interval of several months.

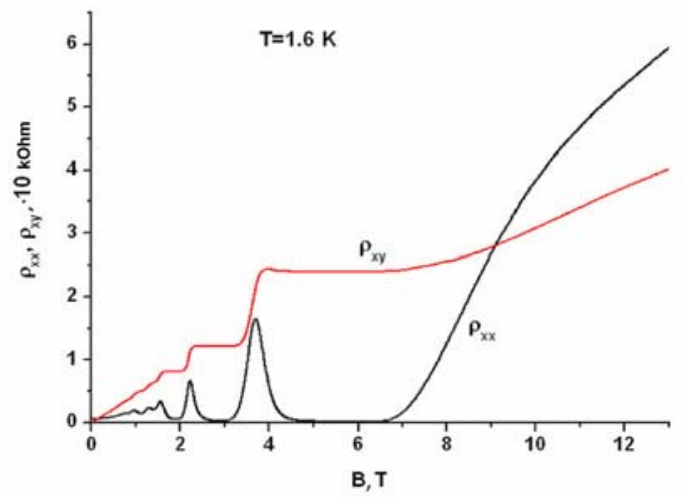

Fig. 5. Longitudinal $\left(\rho_{\mathrm{xx}}\right)$ and Hall $\left(\rho_{\mathrm{xy}}\right)$ components of magnetoresistance versus the magnetic field $(B)$ for 2 DEG in a 21-nm QW.
In the quantum Hall effect regime, the wellpronounced plateaus and minima are observed in all the examined structures. As an example, the longitudinal dissipative $\left(\rho_{\mathrm{xx}}\right)$ and Hall $\left(\rho_{\mathrm{xy}}\right)$ components of magnetoresistance versus the magnetic field $(B)$ are presented in Fig. 5 for the sample N 330. The wide plateaus in $\rho_{x y}(B)$ and the corresponding wide minima in $\rho_{\mathrm{xx}}(\mathrm{B})$ are clearly observed even at $1.6 \mathrm{~K}$. It is due to a small value of the effective mass of electrons in the twodimensional electron gas (2DEG) in a HgTe QW and, respectively, a large distance between Landau levels. The more extensive investigation of the quantum Hall effect in the described samples demonstrates the presence of critical points which are some additional evidence for a high quality of the grown HgTe QW. Such a behavior of magnetoresistances is typical of 2DEG.

\section{Conclusion}

The narrow-gap $\mathrm{Cd}_{\mathrm{x}} \mathrm{Hg}_{1-\mathrm{x}}$ Te-based $(x=0-0.25)$ QWs of $10.5-22 \mathrm{~nm}$ in thickness were grown on GaAs (013) substrates with precise in situ ellipsometric control over the MCT composition and thickness. The determination accuracies of the MCT composition and thickness were $\sim \pm 0.002$ mol. frac. and $\Delta d \sim 0.5 \mathrm{~nm}$, respectively.

The measurements of reflection spectra with layerby-layer chemical etching qualitatively confirmed the results of in situ ellipsometric measurements.

We have demonstrated experimentally the reproducible process of growth of $\mathrm{HgCdTe}$-based QWs.

We have obtained 2DEG with high values of the electron mobility $\mu_{e}=(2.4-3.5) \times 10^{5} \mathrm{~cm}^{2} /(\mathrm{V} \cdot \mathrm{s})$ for concentrations $N_{s}=(1.5-3) \times 10^{11} \mathrm{~cm}^{-2}$ observed for all the grown QW structures, which indicates a high quality of 
QW. The width of dissipative resistance minima and the extensive Hall quantization plateaus testify to a low effective mass of electrons in 2DEG.

\section{Acknowlegements}

The work is partially supported by a complex integration project of SB RAS N 3.20.

The authors are very grateful to L.D. Burdina for carrying out the GaAs substrate preparation and chemical layer-by-layer etching, V.A. Kartashov and I.N. Uzhakov for the growth of buffer layers, and T.I. Zakharyash for the fabricaton of Hall structures.

\section{References}

1. J.N. Schulman, T.C. McGill, The CdTe/HgTe superlattice: Proposal for a new infrared material // Appl. Phys. Lett. 34, p. 663-665 (1979).

2. M.W. Goodwin, M.A. Kinch, R.J. Koestner, Metal-insulator-semiconductor properties of HgTe-CdTe superlattices // J. Vac. Sci. Technol. A 6, p. 2685-2692 (1988).

3. Y.D. Zhou, C.R. Becker, Y. Selament, Y. Chang, R. Ashokan, R.T. Boreiko, T. Aoki, D.J. Smith, A.L. Betz, S. Sivananthan, Far-infrared detector based on $\mathrm{HgTe} / \mathrm{HgCdTe}$ superlattices // J. Electron. Mater. 32(7), p. 608-614 (2003).

4. J.P. Zanatta, F. Noel, P. Ballet, N. Hidadach, A. Million, G. Destefanis, E. Mottin, C. Kopp, E. Picard, E. Hadji, HgCdTe MBE material for microcavity light emitters: application to gas detection in the 2-6 $\mu \mathrm{m}$ range// J. Electron. Mater. 32(7), p. 602-607 (2003).

5. C.R. Becker, X.C. Zhang, A. Pfeuffer-Jeschke, K. Ortner, V. Hock, G. Landwehr, L.W. Molenkamp, Very large Rashba spin-orbit splitting in HgTe quantum wells // Journal of Superconductivity: Incorporating Novel Magnetism 16(4), p. 625-634 (2003).

6. G. Landwehr, J. Gerschutz, S. Oehling, A. Pfeuffer-Jeschke, V. Latussek, C.R. Becker, Quantum transport in n-type modulation-doped mercury telluride quantum well // Physica E 6, p. 713-717 (2000).

7. C.R. Becker, X.C. Zhang, K. Ortner, J. Schmidt, A. Pfeuffer-Jeschke, V. Latussek, Y.S. Gui, V. Daumer, J. Liu, H. Buhmann, G. Landwehr, L.W. Molenkamp, MBE growth and characterization of based $\mathrm{Hg}$ compounds and heterostructures // Thin Solid Films 412, p. 129-138 (2002).

8. C.R. Becker, K. Ortner, X.C. Zhang, A. PfeufferJeschke, V. Latussek, Y.S. Gui, V. Daumer, H. Buhmann, G. Landwehr, L.W. Molenkamp, Growth and studies of $\mathrm{Hgl-xCdxTe}$ based low dimensional structures // Physica E 20, p. 436-443 (2004).
9. N.N. Mikhailov, S.V. Rychlitsky, E.V. Spesivtsev, S.A. Dulin, N.I. Nazarov, Yu.G. Sidorov, S.A. Dvoretsky, Integrated analytical equipment for film growth in MBE technology // Mater. Sci. \& Eng. B 80, p. 41-45 (2001).

10. V.S. Varavin, V.V. Vasiliev, S.A. Dvoretsky, N.N. Mikhailov, V.N. Ovsyuk, Yu.G. Sidorov, A.O. Suslyakov, M.V. Yakushev, A.L. Aseev, HgCdTe epilayers on GaAs: growth and devices // Opto-electronics Review 11(2), p. 99-111 (2003).

11. Yu.G. Sidorov, S.A. Dvoretsky, N.N. Mikhailov, M.V. Yakushev, V.S. Varavin, A.P. Antsiferov, Molecular beam epitaxy of $\mathrm{Cd}_{\mathrm{x}} \mathrm{Hg}_{1-\mathrm{x}} \mathrm{Te}$ compounds: technology and equipment // Optical Journal 67(1), p. 39-45 (2000).

12. Y. Demay, J.P. Galliard, P. Medina, In situ spectroscopic ellipsometry of mercury cadmium telluride MBE layers // J. Cryst. Growth 81, p. 97100 (1987).

13. A.V. Rzhanov, K.K. Svitashev, A.S. Mardezhov, V.A. Shvets, In situ superlattices characterization by ellipsometry // Doklady Akademii Nauk 297(3), p. 604-607 (1987) (in Russian).

14. N.N. Mikhailov, R.N. Smirnov, S.A. Dvoretsky, Yu.G. Sidorov, V.A. Shvets, E.V. Spesivtsev, S.V. Rykhlitski, Growth of $\mathrm{Hg}_{1-\mathrm{x}} \mathrm{Cd}_{\mathrm{x}} \mathrm{Te}$ nanostructures by molecular beam epitaxy with ellipsometric control // Intern. J. Nanotechnology 3(1), p. 120-131 (2006).

15. D.G. Ikusov, S.A. Dvoretsky, V.A. Kartashov, V.A. Kolesnikov, N.N. Mikhailov, I.V. Sabinina, Yu.G. Sidorov, Structural perfection and morphology of the CdTe epitaxial layers // Abstracts of the IX Conference on Semiconductor, Dielectric and Magnetic Materials Physics, p. 176 (2005).

16. P.A. Bakhtin, S.A. Dvoretsky, V.S. Varavin, A.P. Korobkin, N.N. Mikhailov, Yu.G. Sidorov, Conductivity and Hall coefficient to magnetic field dependences in $\mathrm{Cd}_{\mathrm{x}} \mathrm{Hg}_{1-\mathrm{x}}$ Te films grown by molecular beam epitaxy // Fizika Tekhnika Poluprovodnikov 38(10), p. 1203-1206 (2004) (in Russian).

17. E.V. Spesivtsev, S.V. Rykhlitski, Useful model "Ellipsometer." License No. 16314 RF // Bulletin "Useful models. Industrial samples" 35 (20.12.2000).

18. R.M.A. Azzam, N.M. Bashara, Ellipsometry and Polarized Light. North-Holland, Amsterdam, 1977.

19. V.A. Shvets, S.V. Rykhlitski, E.V. Spesivtsev, N.A. Aulchenko, N.N. Mikhailov, S.A. Dvoretsky, Yu.G. Sidorov, R.N. Smirnov, In situ ellipsometry for control of $\mathrm{Hg}_{1-\mathrm{x}} \mathrm{Cd}_{\mathrm{x}} \mathrm{Te}$ nanolayer structures and inhomogeneous during MBE growth // Thin Solid Films 455-456, p. 688-694 (2004).

20. K.K. Svitashev, S.A. Dvoretsky, Yu.G. Sidorov, V.A. Shvets et al., The growth of high-quality MCT films by MBE using in situ ellipsometry // Cryst. Res. Technol. 29(7), p. 931-937 (1994). 
21. V.A. Shvets, N.N. Mikhailov, M.V. Yakushev, E.V. Spesivtsev, Ellipsometric measurements of the optical constants of solids under impulse heating // Proc. SPIE 4900, p. 46-52 (2002).

22. H. Arwin, D.E. Aspnes, Nondestructive analysis of $\mathrm{Hg}_{1-\mathrm{x}} \mathrm{Cd}_{\mathrm{x}} \mathrm{Te}(\mathrm{x}=0.00,0.2,0.29$, and 1.00$)$ by spectroscopic ellipsometry. Pt. 2. Substrate, oxide and interface properties // J. Vac. Sci. Technol. A 2, p. 1316-1323 (1984).

23. L. Vina, C. Umbach, M. Cardona, L. Vodopjanov, Ellipsometric studies of electronic interband transitions in $\mathrm{Cd}_{\mathrm{x}} \mathrm{Hg}_{1-\mathrm{x}} \mathrm{Te} / /$ Phys. Rev. B 29, p. 6752-6760 (1984).

24. N.A. Kornyushkin, T.I. Kurdina, A.S. Mardezhov et al., Optical and electrical properties of $\mathrm{HgTe}$ films grown by molecular beam epitaxy // Poverkhnost'. Fizika, khimiya, mekhanika 8, p. 94100 (1991) (in Russian).

25. L. Vina, C. Umbach, M. Cardona, L. Vodopyanov, Ellipsometric studies of electronic interband transitions in $\mathrm{Cd}_{\mathrm{x}} \mathrm{Hg}_{1-\mathrm{x}} \mathrm{Te} / /$ Phys. Rev. B 29(12), p. $6752-6760$ (1984). 